\title{
Risk factors in repeat non-fatal suicidal behaviour
}

The risk of repeating a suicidal act is a critical characteristic of the suicidal process. Research' has shown that in fatal suicidal behaviour up to $40 \%$ of the victims have a history of previous suicide attempts; that in non-fatal suicidal behaviour in some patient cohorts, repeated suicidal acts are probably more common than in first attempts; that $30-60 \%$ of patients with non-fatal suicidal behaviour have made previous attempts, in $15-25 \%$ of cases within the first year before the suicidal act; and that prospectively, non-fatal suicidal behaviour results in a high risk of future fatal suicidal behaviour (between 10\% and $15 \%$ of patients may eventually succeed in committing suicide, with the risk of repeat suicidal behaviour being highest during the first year after a suicide attempt - especially within the first 3 - 6 months). However, the incidence and prevalence rates vary significantly between countries and different cultures.

Accordingly, there is an obvious need for information on how to predict suicidal behaviour and prevent repetitions of non-fatal suicidal behaviour. A body of international research ${ }^{1-2}$ has found risk factors associated with a high risk of such repetition to include the age group 25 - 49 years; divorce; unemployment; lower socioeconomic groups; hopelessness; powerlessness; substance abuse; depression; personality disorders; unstable living conditions; living alone; a criminal record; a history of previous psychiatric treatment; stressful/traumatic life events; family violence; and previous non-fatal suicidal behaviour. Research ${ }^{3}$ emphasises that this latter point is one of the most valid measurements to predict repeat suicidal behaviour, along with social isolation, which can be seen as analogous to a history of previous episodes of depression being a powerful predictor of future onset of depression. Nevertheless, various psychometric scales have been developed to assist with suicidal behaviour risk assessment, ${ }^{3}$ such as the Zung Index of Potential Suicide, the Risk Estimator of Suicide, the Risk of Repetition Scale, the Post Attempt Risk Assessment Scale, the Scale of Suicide Ideation developed by Beck and his co-workers, the Beck Hopelessness Scale, and the SAD PERSONS Scale for assessing the risk of suicide. Such scales identify various domains of suicidality and vary in their reliability, validity and clinical usefulness. Space does not allow for an in-depth discussion of such issues here, save to note that it is desirable that apart from being valid and reliable any scale used in clinical practice should be simple and brief, preferably with the number of items being 10 or less. ${ }^{3}$

Such considerations assume even greater importance if we consider that suicidal behaviour is a serious problem globally and in South Africa. ${ }^{45}$ Annually approximately 1 million people worldwide commit suicide, a figure that is likely to increase to about 1.5 million per annum by 2020. ${ }^{4}$ About 10 to 20 times more non-fatal than fatal suicides occur, and this could be as high as 40 times more in some regions of the world. These figures give a rate of 16 per 100000 of the world population and represent 1 death every 40 seconds and 1 attempt every 3 seconds worldwide. Between 1950 and 1995 there has been almost a $60 \%$ increase worldwide in fatal suicidal behaviour. ${ }^{45}$

The rate of suicidal behaviour in South Africa is just as inordinately high, and past (Apartheid-era) data generally recorded a bias with regard to the real dimension of the burden of suicidal behaviour in certain South African communities because of under-reporting, especially in black South Africans. ${ }^{5}$ Likewise, figures on suicidal behaviour in the rest of Africa are not that readily available. ${ }^{5}$ This complicates an accurate analysis of prevalence rates of suicidal behaviour in Africa and South Africa and places limitations on interpreting clinical and epidemiological trends. Nevertheless, general trend analyses are possible, ${ }^{5}$ given research findings since the advent of democracy in South Africa. For example, an increase in suicidal behaviour of up to $48 \%$ has been observed in black South Africans in some areas during the last few years. ${ }^{5}$ The most recent figures ${ }^{5}$ show that suicide accounted for up to $11 \%$ of all non-natural deaths in South Africa, and that non-fatal suicidal behaviour in general hospitals accounted for up to 12\% of patients referred for psychological/psychiatric help. It can be estimated that one South African dies of suicide every hour, and that 20 - 40 non-fatal suicides occur per hour. ${ }^{5}$ A significant proportion of these are repeat suicidal behaviours.

Because suicidal behaviour is a process it constitutes a complex phenomenon that cannot readily be attributed to any single cause as it involves intricate interactions between psychological, social, cultural and biological variables, although as in the case of international findings, specific risk factors and critical aetiological considerations in South Africans have been identified. This is also true in the case of repeat suicidal behaviours. In line with findings on the risk factors for repeat suicidal behaviour, South African research ${ }^{5}$ has echoed time and again that risk factors associated with suicidal behaviour include family psychopathology (such as a history of family members' suicidal behaviour, substance abuse and other psychological disorders), academic problems in young people, reading impairment (which is associated with one of the most prominently investigated learning disabilities internationally and can be a co-morbid factor in suicidal behaviour, especially in young people, in whom it can contribute to certain psychopathological disorders), exposure to family violence, fundamentalism and fanatical belief systems, child abuse, bereavement, interpersonal problems (especially marital, partner-relational and family problems), financial problems, psychiatric disorders such as depression and substance abuse (especially alcohol abuse), personality 
disorders, a history of sexual abuse, prior suicidal behaviour in the index patient, socioeconomic difficulties including high unemployment levels and high prevalence rates of violence and trauma, and dysfunctional perceptions in undue stress arousal associated with suicidal behaviour.

Additional risk factors include findings related to the neurobiology of suicidal behaviour, ${ }^{6}$ such as, for example, a common biological substrate underlying the propensity for suicidal behaviour associated with violence, aggression, impulsivity and stress; neurological findings that show a correlation between altered serotonergic activity and suicidal behaviour; genetic factors; abnormalities in the serotonergic (5hydroxytryptamine, 5-HT) system and in other neurotransmitter systems, such as the noradrenergic, dopaminergic, GABA-ergic and glutamatergic systems; and hyperactivity of the hypothalamic-pituitary-adrenal axis. These factors also need to be taken into account in an understanding of repeat suicidal behaviours (both fatal and non-fatal).

Further, images produced by sophisticated brain-scanning techniques can reveal brain abnormalities and help solve some of the mysteries of human behaviour associated with various problems such as depression and suicidal behaviour. With regard to suicidal behaviour, several promising lines of research are underway and even more benefits of neuroimaging technology may result as researchers combine the procedures with other techniques, such as genetic or biochemical markers of suicidal behaviour. Increasingly, technical advances in neuroimaging allow for the localisation and analysis of specific chemical and electrical activities within the human brain during normal and abnormal mental processes lincluding during suicidal behaviour). ${ }^{7}$ Findings ${ }^{8}$ from this field of functional neuroimaging have suggested the role of particular brain areas in the psychological or biological predisposition to suicidal behaviour. These studies have revealed a strong body of evidence of the role of various neurobiological systems in the pathophysiology of suicidal behaviour, i.e. hyperactivity of the hypothalamo-pituitary-adrenal axis, a dysfunction of the serotonergic system and an excessive activity of the noradrenergic system. Results of studies ${ }^{8}$ have pointed to dysfunction of the serotonergic system in association with feelings of hopelessness and disturbances in the regulation of anxiety, impulsivity and/or aggression, which may be due to genetic or non-genetic familial and/or to environmental factors (e.g. a dysfunctional or traumatic childhood). Regarding the latter issue, it has been found that children who grow up with negative cognitive schemata are at greater risk for depression and suicidal behaviour in later years compared with controls. ${ }^{5}$

There is a disconcerting 'ungreying' phenomenon in suicidal behaviour both globally ${ }^{4}$ (including repeat incidents) and in South Africa. ${ }^{5}$ The average age for fatal suicidal behaviour in South Africa is now around 35 years and a national mean age of 25 years for non-fatal suicidal behaviour in general hospital samples has been reported, with patients as young as 6 years old who make attempts and patients as young as 10 years who succeed in committing suicide. This trend is consistent with the global trend ${ }^{4}$ of a shift from suicide in the elderly towards younger people.

South African research ${ }^{5}$ has shown that in general, the choice of method used in suicidal behaviour for all age groups is strongly influenced by factors such as: (i) access to the method; (ii) knowledge of the lethality or otherwise of the method; (iii) experience and familiarity with the method; (iv) the meaning, symbolism and cultural influence associated with the method; and $(v)$ the potential suicidal person's state of mind and level of intent at the time of the suicidal act. Further, ${ }^{5}$ not only are suicidal patients often poor at solving interpersonal problems but as noted, chronic and acute stress are critical co-morbid aetiological risk factors in suicidal behaviour. These concerns are crucial considerations in repeat non-fatal suicidal behaviour because with repeated acts of such behaviour: (i) more severe and more lethal methods may be used; and (ii) elevated recklessness and impulsiveness ensue if the ongoing non-fatal suicidal behaviour does not get the desired response from significant others on whom it is supposed to impact, resulting in a further increase in repeat suicidal behaviour.

I have stated before that to improve this situation we need to recognise that the prediction, prevention and management of suicidal behaviour should be everyone's responsibility and not only that of mental health specialists. This is particularly important in repeat non-fatal suicidal behaviour, which continues to be a major prevention challenge despite the effect of combined or sequential pharmacological, psychotherapeutic and other interventions. Further South African research is clearly required, and the article by Moosa et al. ${ }^{9}$ on repeat non-fatal suicidal behaviour in this issue of the South African Journal of Psychiatry is a much needed contribution.

\section{Lourens Schlebusch}

\section{Department of Behavioural Medicine Nelson R Mandela School of Medicine University of KwaZulu-Natal Durban}

1. Kerkhof AIFM. Attempted suicide: patterns and trends. In: Hawton K, van Heeringen K, eds. The International Handbook of Suicide and Attempted Suicide. Chichester: John Wiley, 2000: 49-64.

2. Goldney RD. Prediction of suicide and attempted suicide. In: Hawton K, van Heeringen, K, eds. The International Handbook of Suicide and Attempted Suicide. Chichester: John Wiley, 2000: 585-595

3. Bech P, Olsen R, Niméus A. Psychometric scales in suicide risk assessment. In: Wasserman D, ed. Suicide: An Unnecessary Death. London: Martin Dunitz, 2001 : 147-157.

4. Bertolote JM. Suicide in the world: An epidemiological overview 1959-2000. In: Wasserman D, ed. Suicide: An Unnecessary Death. London: Martin Dunitz, 2001: 3 10 .

5. Schlebusch L. Suicidal Behaviour in South Africa. Pietermaritzburg: University of KwaZulu-Natal Press, 2005

6. Träskman-Bendz L, Mann $\downarrow$. Biological aspects of suicidal behaviour. In: Hawton K van Heeringen K, eds. The International Handbook of Suicide and Attempted Suicide. Chichester: John Wiley, 2000: 65-77.

7. Kaplan HI, Sadock BJ. Synopsis of Psychiatry Behavioural Sciences/Clinical Psychiatry Baltimore, Md: Lippincott Williams \& Wilkins, 1998

8. Van Heeringen K. Neurobiology and genetics of suicide. Paper presented at the XXIII World Congress of the International Association for Suicide Prevention, Durban, 13-16 September 2005

9. Moosa MYH, Jeenah FY, Vorster M. Repeat non-fatal suicidal behaviour at Johannesburg Hospital. South African Journal of Psychiatry 2005; 11: 84-88 (his issue). 